\title{
Development of chronic conjunctivitis with scarring and pannus, resembling trachoma, in guinea-pigs
}

\author{
MARJORIE A. MONNICKENDAM, S. DAROUGAR, \\ J. D. TREHARNE, AND ANGELA M. TILBURY \\ From the Virus Laboratory, Department of Clinical Ophthalmology, Institute of Ophthalmology, \\ Judd Street, London WCIH $9 Q S$
}

SUMMARY Guinea-pigs were repeatedly infected with guinea-pig inclusion conjunctivitis agent. Reinfection caused severe conjunctival inflammation, and repeated reinfection led to chronic inflammation lasting for many months. This was followed by the development of pannus, follicles on the palpebral conjunctivae, scarring of the lower palpebral conjunctiva, and deformities of the lower lid. Reinfection was accompanied by small numbers of inclusion-bearing cells, small numbers of polymorphonuclear cells, and high numbers of mononuclear cells. There was no increase in the level of serum antibodies. The chronic conjunctivitis was associated with high numbers of mononuclear cells and no inclusions or polymorphonuclear cells. The response to reinfection appears to be a delayed-type hypersensitivity reaction, and we suggest that the chronic inflammation, pannus, scarring, and lid deformities associated with hyperendemic trachoma may be due to repeated reinfection combined with delayed-type hypersensitivity.

Primary ocular infection with guinea-pig inclusion conjunctivitis (GPIC) agent produces acute conjunctivitis, with no pannus or scarring, which resolves spontaneously in 30 to 40 days. ${ }^{1-3}$ It has not therefore been considered a good model for trachoma.

In this study we have looked at the ocular responses of guinea-pigs to reinfection with GPIC agent, and compared them with the responses to primary infection. We have found that repeated reinfection produces chronic conjunctivitis with scarring and pannus, similar to trachoma.

\section{Materials and methods}

Animals, organisms, methods for clinical assessment, conjunctival scrapings and serology are described in the previous paper. ${ }^{3}$

EXPERIMENTAL DESIGN

Animals were infected and reinfected in the left eye, with 1 drop of inoculum (containing $10^{6} \mathrm{EID}_{50}$ of GPIC agent strain A10) as described in the previous paper. ${ }^{3}$

The first experiment was carried out on 5 fullygrown animals, which were examined, photographed

Correspondence to Dr M. A. Monnickendam. and scraped immediately before infection and at intervals after infection and reinfection. These animals were infected 7 times.

The second experiment was carried out on 128 young guinea-pigs. Fresh groups of 4 animals were taken immediately before infection and at various intervals after infection and reinfection; eyes were examined, photographed and scraped, and blood collected for serology. These animals were infected 5 times.

\section{Results}

DURATION AND SEVERITY OF DISEASE In the first experiment the duration of conjunctival inflammation increased in successive infections (Fig. 1d). After the seventh infection inflammation persisted for over a year.

In the second experiment (Fig. $2 e$ ) infections were carried out at 35- and 28-day intervals, when inflammation from previous infections was still present.

In both experiments the severity of inflammation was generally greater during reinfection (Figs. 1d, $2 e$ ). In the early reinfections there was acute, severe inflammation lasting for 4 to 7 days (Fig. 5), followed by a mild to moderate inflammation, lasting several weeks or months. In later infections the 
Figure la
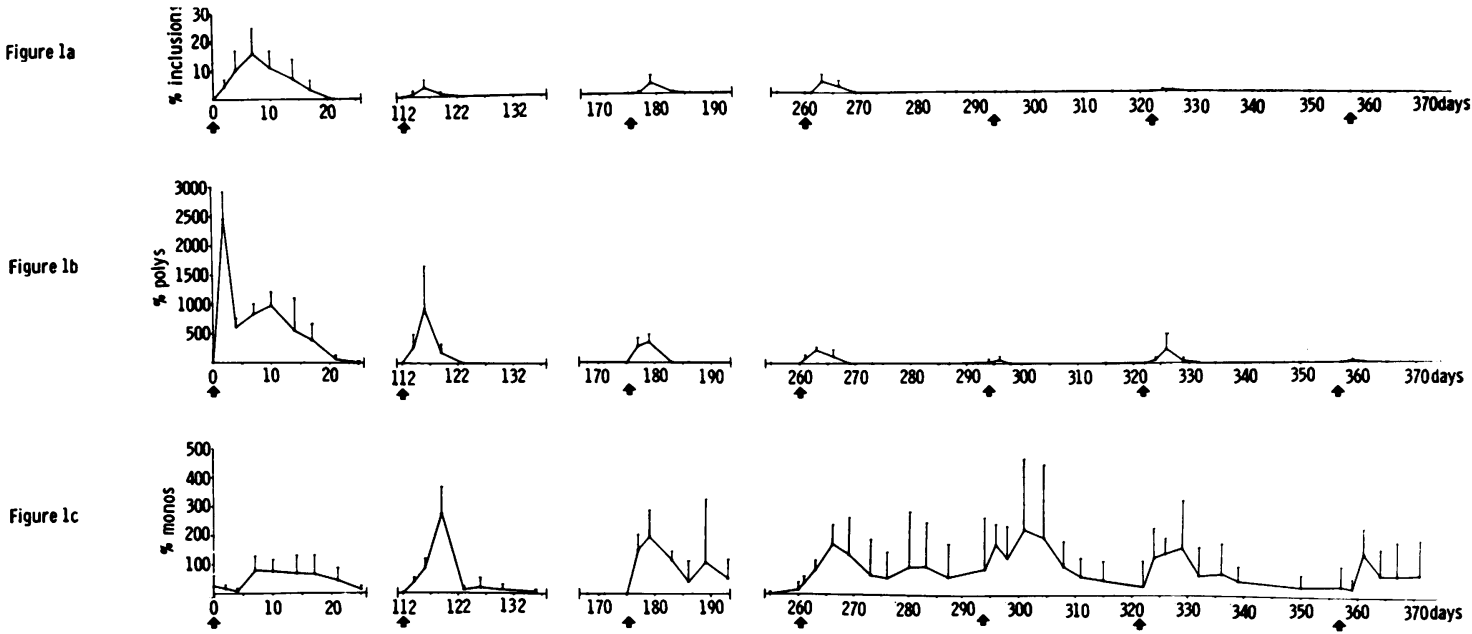

Figure ld
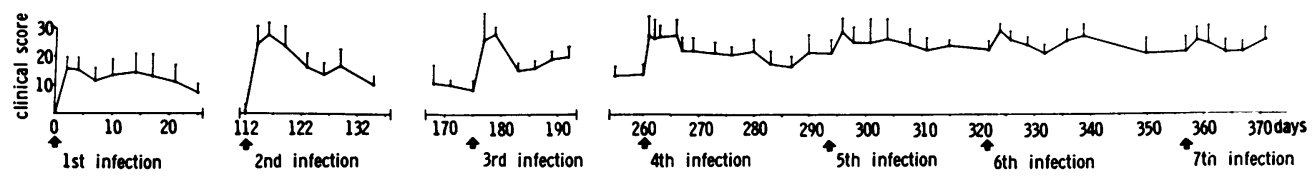

Fig. 1 The responses of 5 animals to 7 successive GPIC agent infections (mean + standard deviation). Arrows indicate inoculations.

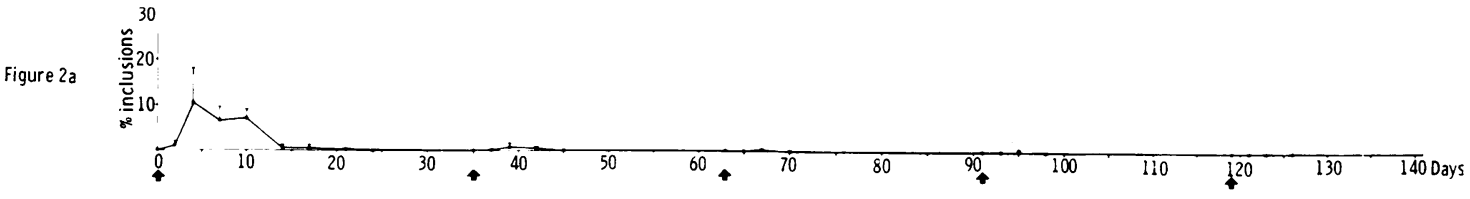
Figure 2b $\frac{2000}{\bar{c}} 500$
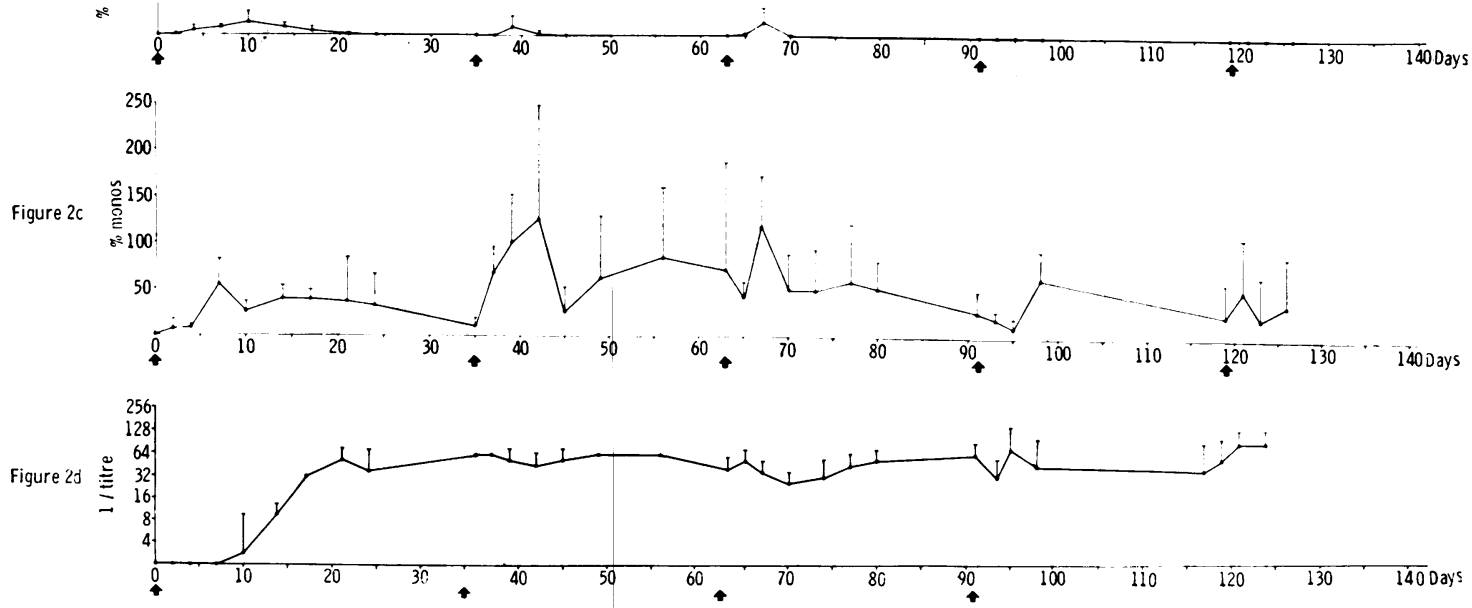

Figure $2 \epsilon$

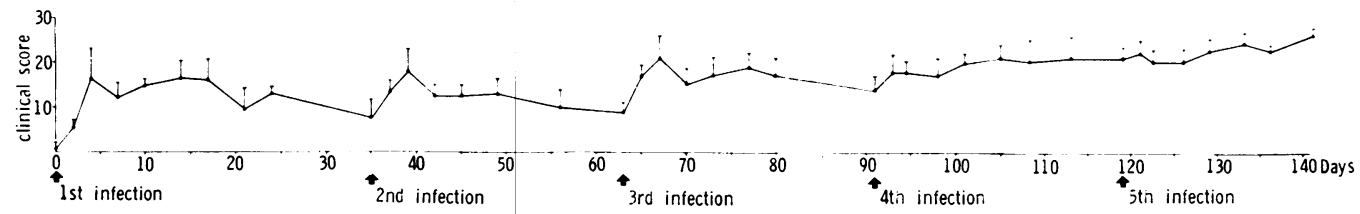

Fig. 2 The responses of groups of 4 animals, which had not previously been scraped, to 5 successive GPIC agent infections (mean + standard deviation). Arrows indicate inoculations. 
Figure 30

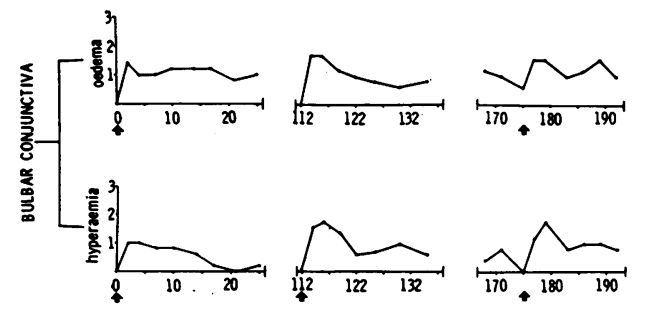

Figure 3b
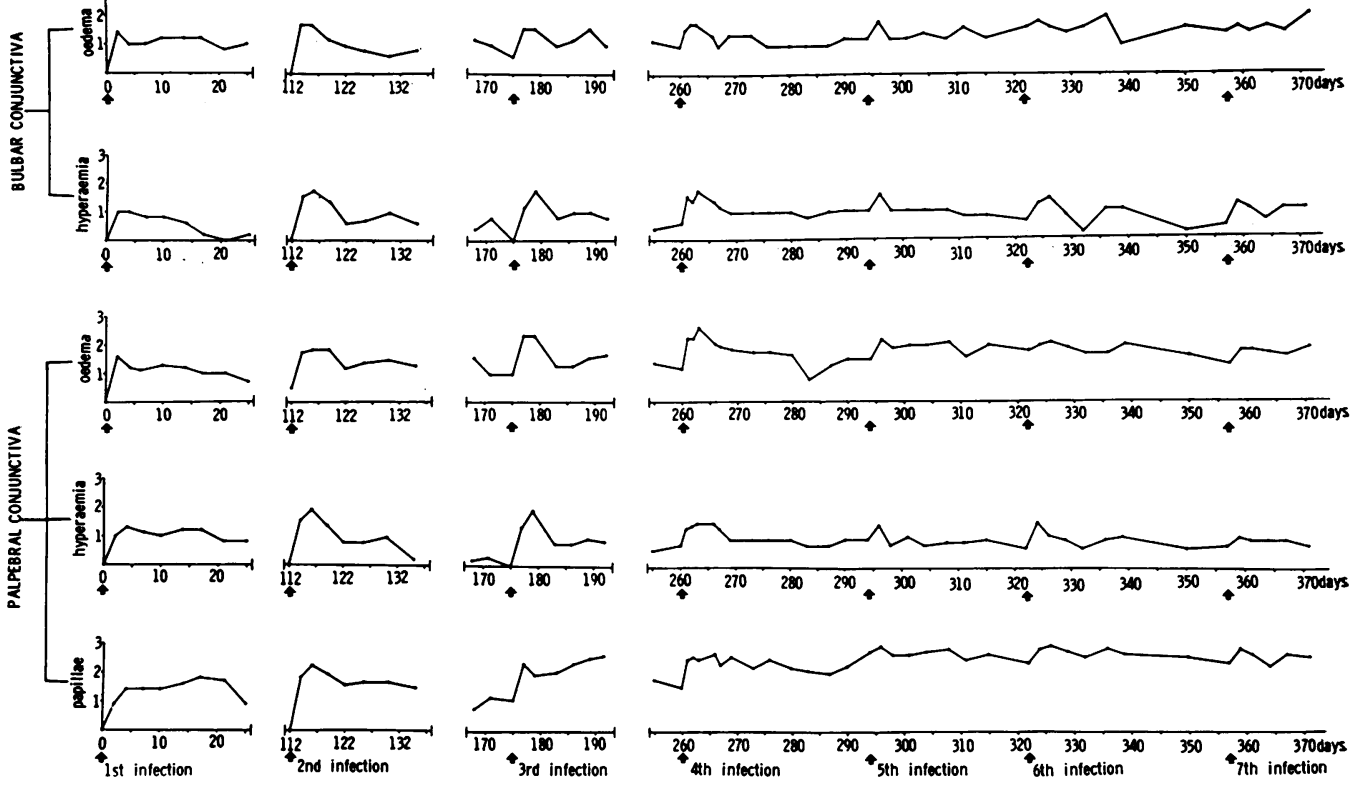

figure $3 c$

Figure 30

Figure 3.
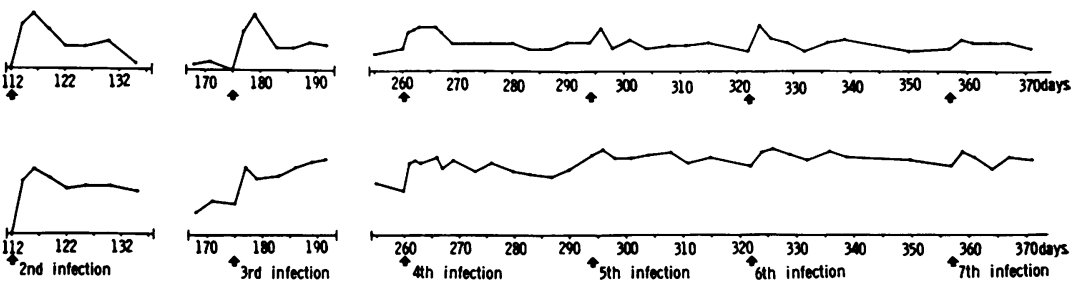

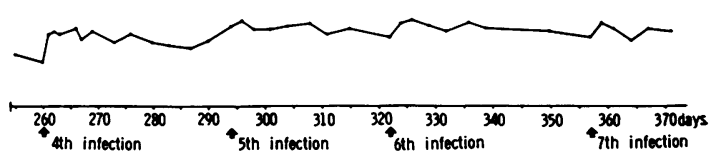

Fig. 3 The intensity and duration of signs in 5 animals to 7 successive GPIC infections. Arrows indicate inoculations.

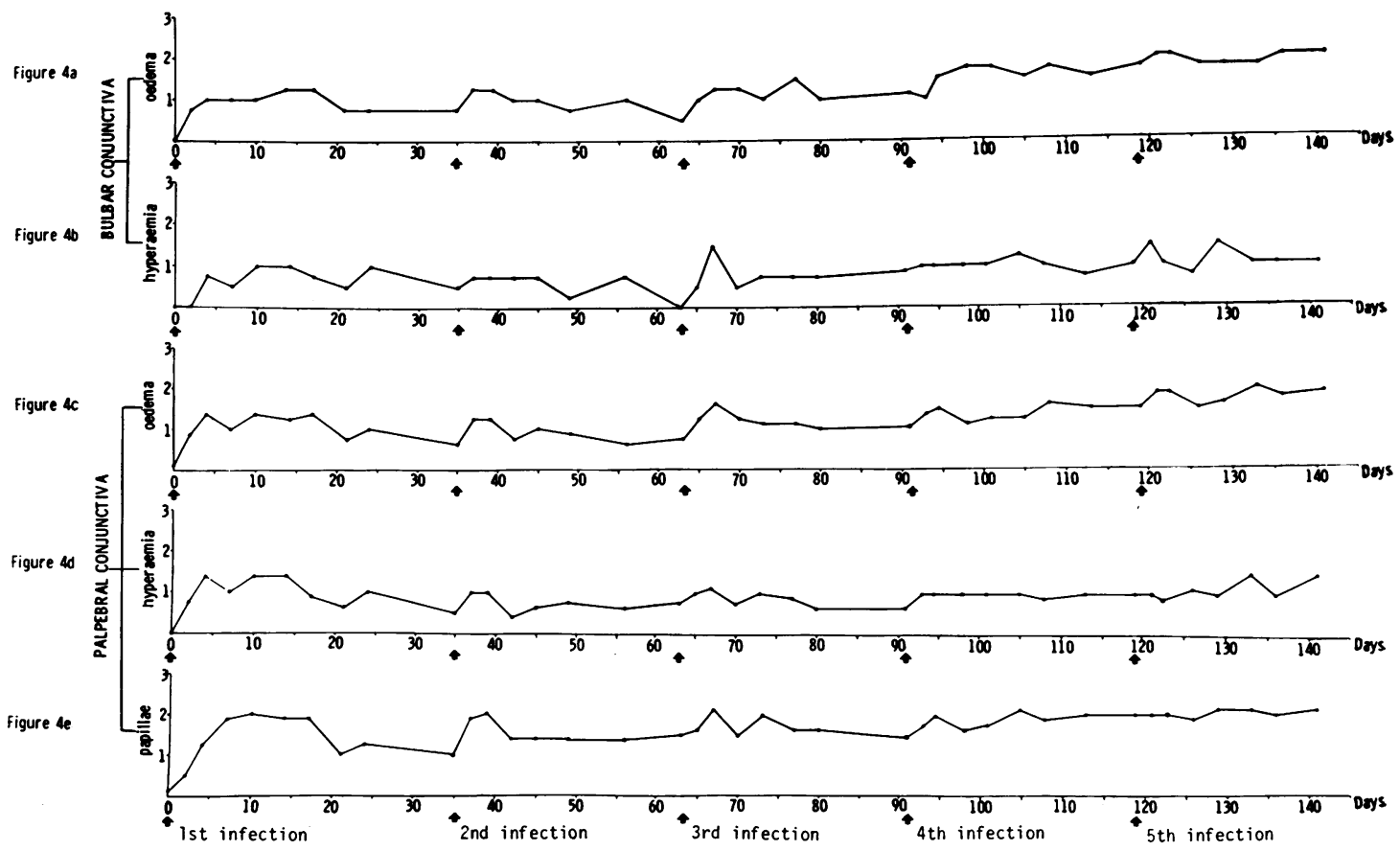

Fig. 4 The intensity and duration of signs in groups of 4 animals, which had not previously been scraped, to 5 successive GPIC agent infections. Arrows indicate inoculations. 


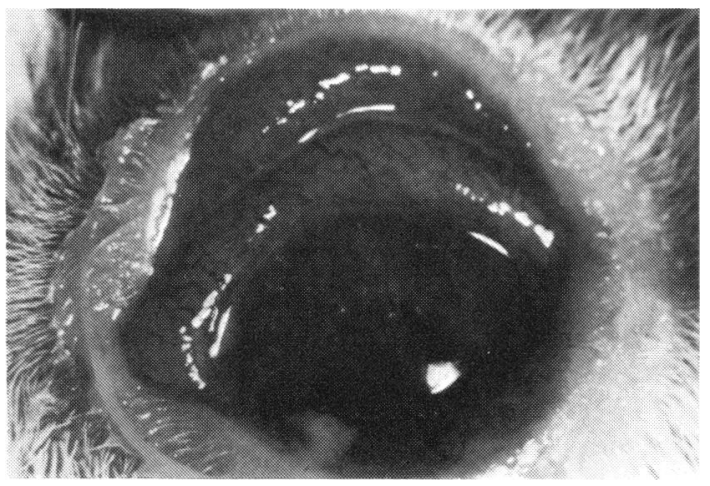

Fig. 5 Inflammatory responses 4 days after the second GPIC agent infection showing severe hyperaemia and oedema of the upper palpebral and bulbar conjunctivae.

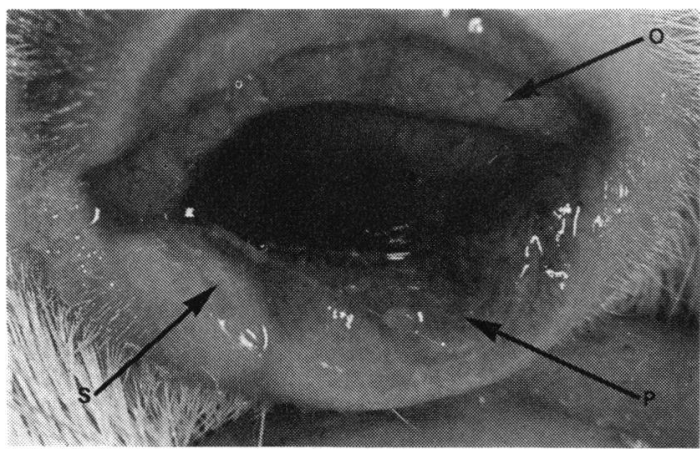

Fig. 6 Inflammatory responses 4 days after the seventh GPIC agent infection showing oedema of the bulbar (o), diffuse infiltration, severe papillary reaction $(p)$ and scarring $(s)$.

severity of the chronic inflammation resulting from previous infections was so great that very little, if any, increase was observed on subsequent inoculation.

\section{SIGNS}

The clinical signs were very similar in the 2 experiments. Lacrimation and discharge, and erythema and oedema of the lids were frequently observed for up to 7 days after reinfection but were rarely seen at other times.

The changes in the intensity of bulbar oedema and hyperaemia, palpebral oedema, hyperaemia, and papillae are shown in Figs. 3 and 4. Bulbar oedema tended to become progressively more severe in successive infections (Figs. $3 a, 4 a$ ), particularly in the second experiment. Bulbar hyperaemia tended to be less severe, particularly in later infections (Figs. 3b, 4b, 5, 6).

In the palpebral conjunctivae the severity of hyperaemia, oedema, and papillary hypertrophy were the same in upper and lower lids. The changes in the intensity of oedema and hyperaemia were similar to those seen in the bulbar conjunctiva (Figs. $3 c, 3 d, 4 c, 4 d, 5,6$ ). Papillary hypertrophy became more severe in successive infections (Figs. $3 e, 4 e$ ).

No follicles were seen on the palpebral conjunctivae during primary infection, though the conjunctival folds, which became swollen during infection, looked similar to follicles. True follicles appeared during subsequent infections. A few small follicles were seen on both the upper and lower palpebral conjunctivae 7 to 10 days after the second infection; these soon disappeared. More follicles appeared during subsequent infections; these were larger and persisted for longer (Fig. 7). During later infections many large follicles were present on the upper palpebral conjunctiva, whereas progressively fewer and smaller follicles were found on the lower palpebral conjunctiva.

Small diffuse scars were observed on the lower palpebral conjunctiva of 3 animals used in the first

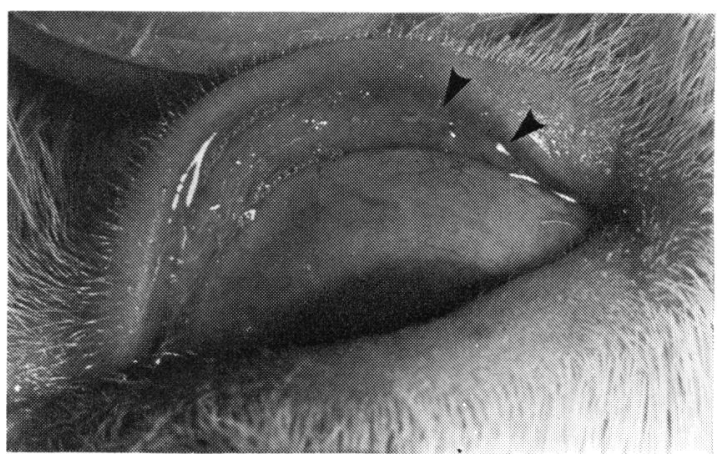

Fig. 7 The upper palpebral conjunctiva showing follicles.

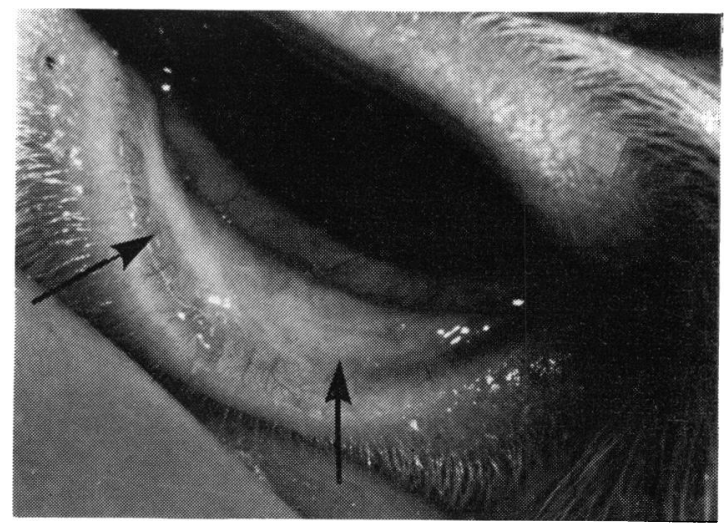

Fig. 8 The lower palpebral conjunctiva showing diffuse scarring. 


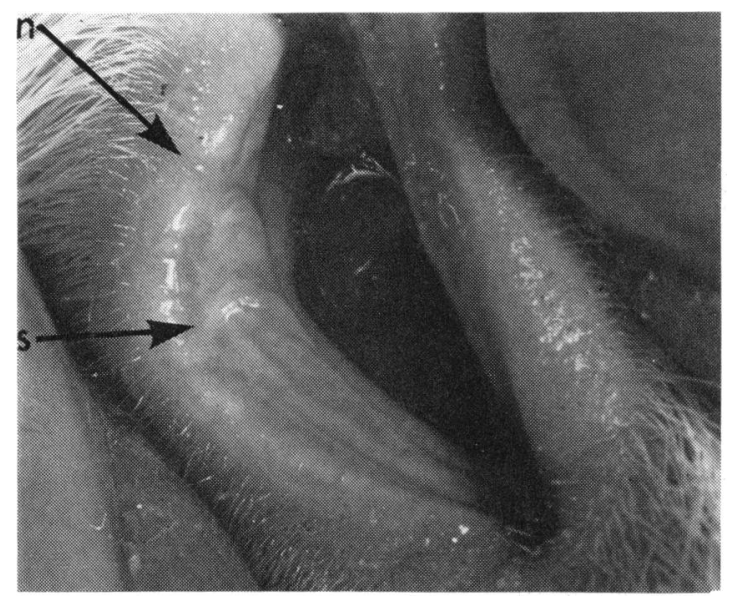

Fig. 9 The lower palpebral conjunctiva showing synechial scarring (s) and notch $(n)$ at the lid margin.

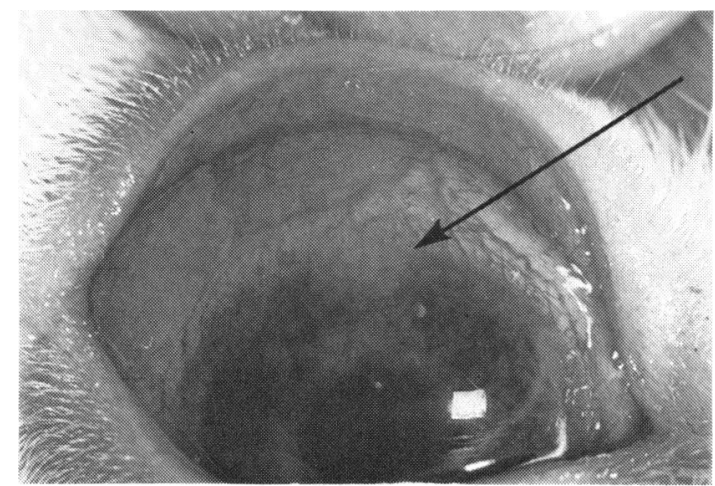

Fig. 10 Marginal diffuse keratitis.

experiment 65 days after the third infection. All 5 animals had developed diffuse scars 34 days after the fourth infection. The scarring became more severe during successive infections, and linear and synechial scars also developed (Figs. 8 and 9). In the second experiment, small diffuse scars were first seen on the lower palpebral conjunctivae of all 4 animals examined 4 days after the fourth infection. Larger diffuse scars were seen in animals during the fifth infection, and linear and synechial scars were occasionally seen.

In the cornea a mild diffuse epithelial and subepithelial keratitis was commonly observed for up to 7 days following the second infection (Fig. 10) and showed no increase in severity in successive infections. Neovascularisation associated with infiltration (active pannus) was detected in the upper region of the cornea. In the first experiment it was first observed 10 to 14 days after the fifth infection and was present in all 5 animals 3 months after the seventh infection. In the second experiment it was first observed several weeks after the fifth infection (Figs. 11 and 12).

Deformities of the lower lids developed in 1 animal used in the first experiment. These were first observed several months after the seventh infection. A notch developed on the left lower lid which was associated with synechial scarring (Figs. 6 and 9), and entropion of the right lower lid, which was associated with heavy scarring (Figs. 8 and 13). In the second experiment entropion of the lower lid was observed in 6 eyes from 15 animals examined 12 months after the fifth infection. Deformities of the upper lid were not seen.

The right (uninoculated) eye also developed clinical signs of disease. These were similar to those seen in the inoculated eye, but were less severe, reaching a maximum 7 to 14 days after inoculation of the left eye. Chronic conjunctivitis, associated with follicles and scarring of the palpebral conjunctiva, and pannus also developed in the right eye.

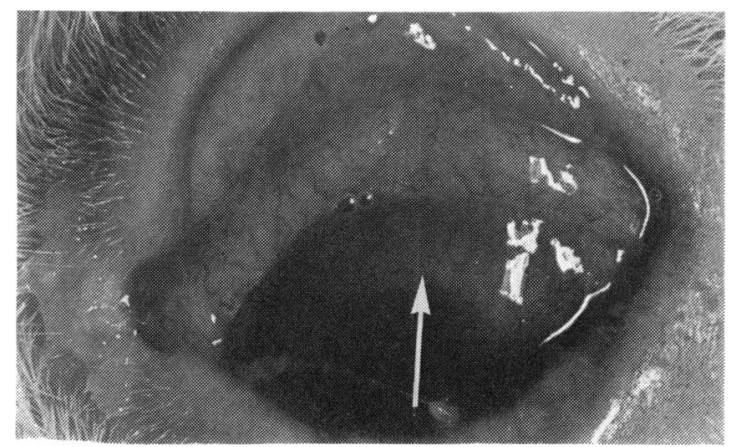

Fig. 11 Early pannus.

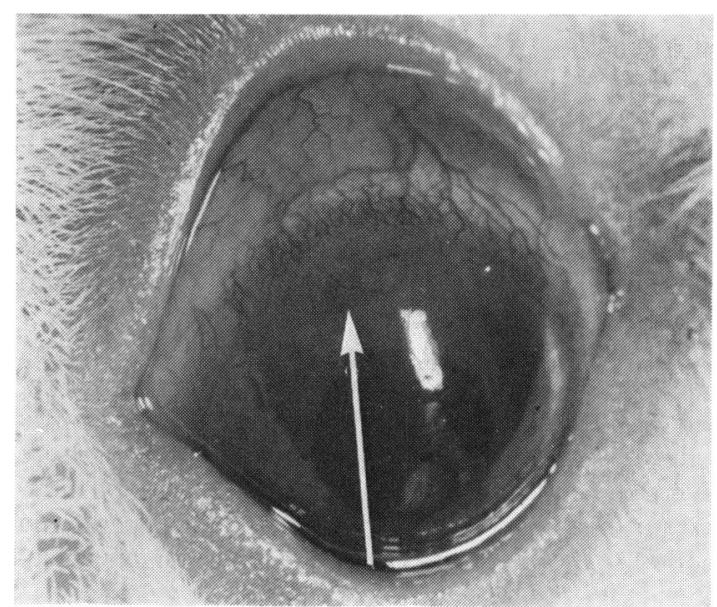

Fig. 12 Severe pannus with diffuse corneal infiltration. 


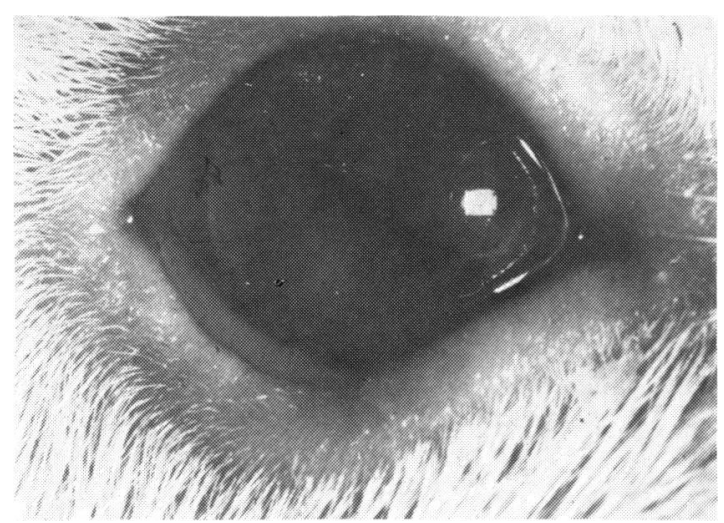

Fig. 13 Mild entropion of the lower lid associated with diffuse scarring (shown in Fig. 8).

\section{CHLAMYDIAL INCLUSIONS}

In both experiments, many inclusions were seen from days 2 to 21 after primary infection. The percentage of epithelial cells containing inclusions was greatly reduced in reinfection (Figs. $1 a$ and $2 a$ ). In the first experiment inclusions were seen in scrapings from all 5 animals for up to 7 days after the second, third, and fourth infections, a few inclusions were seen in 2 out of 5 animals after the sixth infection; none were seen after the fifth and seventh infections (Fig. 1a). In the second experiment a few inclusions were seen in some animals 2 and 4 days after the second, third, and fourth infections; no inclusions were seen following the fifth infection (Fig. 2a).

\section{POLYMORPHONUCLEAR CELLS}

In the first experiment large numbers of polymorphonuclear cells were seen on days 2 to 17 following primary infection. In subsequent infections the number of cells was greatly reduced (Fig. 1b). In the second experiment far fewer cells were seen in the primary infection, and the numbers were further reduced in subsequent infections (Fig. 2b).

Most of the polymorphonuclear cells seen after reinfection were neutrophils, but a few eosinophils and basophils were also present.

\section{MONONUCLEAR CELLS}

In both experiments raised levels of mononuclear cells were seen from days 7 to 24 after primary infection (Figs. $1 c$ and $2 c$ ). In the first experiment the number of cells seen in the 7 days following reinoculation was much higher except for the seventh infection. After the initial 7 days animals varied. Some had normal levels, and others had persistently raised levels of cells, so that no further increase was seen on reinoculation. No consistent pattern of response followed the seventh infection (Fig. 1c).

In the second experiment raised levels of mononuclear cells were seen for 7 days after the second infection; after this some animals had high levels of mononuclear cells, and others had low levels. No consistent pattern of response was seen in the third, fourth, and fifth infections (Fig. $2 c$ ).

\section{SERUM ANTIBODIES}

Total serum antibodies against GPIC agent were measured in the animals used in the second experiment. Antibodies were first detected 10 days after primary infection and reached a peak on day 21 . There was no significant change in the level of serum antibodies after 4 more infections (Fig. 2d).

\section{Discussion}

Repeated reinfection of guinea-pig eyes with GPIC agent produced chronic inflammation and lesions similar to those of trachoma. The microbiological and cytological responses to reinfection were very different from those seen in primary infection. Small numbers of inclusions were found for up to 7 days after reinfection and sometimes none were seen. This confirms previous observations that guinea-pigs became partially immune to GPIC agent after primary ocular infection. ${ }^{1245}$ Similar observations have been made in owl monkeys and human volunteers infected with Chlamydia trachomatis strains isolated from patients with ophthalmia neonatorum or trachoma. ${ }^{6} 7$

In our experiments the degree of immunity depended on the time interval between infections. When it was greater than 60 days, inclusions were seen in all animals for up to 7 days after reinfection (Fig. 1a). When it was reduced to 35 days or less, inclusions were only occasionally seen. Sometimes none were found (Figs. $1 a$ and $2 a$ ). Fewer polymorphonuclear cells were seen during reinfection than in primary infection. They were found only when inclusions were present. Many more mononuclear cells were found than in primary infection. In the second infection of both experiments large numbers were present when inclusions were found, but in later infections high levels of mononuclear cells persisted for long periods when there were no inclusions. No increase in the levels of serum antibodies was seen after reinfection.

How is the reduction in the number of inclusions in reinfection mediated? A study of the role of humoral immunity has shown that in guinea-pigs the transfer of immune serum before primary ocular infection does not result in a reduction of the number of inclusions. ${ }^{8}$ Since we found increased numbers of mononuclear cells in the eye during reinfection, we 
would suggest that they may mediate this reduction.

In guinea-pigs which have had an ocular GPIC agent infection a delayed-type hypersensitivity skin reaction is observed when live GPIC agent is administered intradermally, ${ }^{9}$ and we have observed large numbers of mononuclear cells at the sites of such skin reactions (unpublished results). Since ocular administration of live GPIC agent to previously infected animals produced severe inflammation accompanied by increased numbers of mononuclear cells, it seems reasonable to consider this reaction as an ocular delayed-type hypersensitivity reaction analogous to the skin reaction. Both the ocular and skin reactions could be elicited long after sensitisation-the skin reaction for at least 2 years after primary ocular infection ${ }^{9}$ and the eye reaction for at least 1 year after a previous ocular challenge. Uninfected yolk material did not produce dermal or ocular reactions (unpublished results).

Since chronic conjunctivitis developed in the animals used in both experiments, in the first of which animals were repeatedly scraped and in the second of which animals were used on 1 occasion only and scraped after clinical examination, we suggest that trauma plays little, if any, role in the production of chronic disease in guinea-pigs. Hanna $^{10}$ found that, when American Indians with trachoma were subjected to two conjunctival scrapings at an interval of about 7 days, the number of inclusion-positive scrapings was significantly higher in the second set of specimens. We found inclusions in guinea-pigs with chronic conjunctivitis only within 7 days of reinfection, and repeated scraping did not increase the number of inclusions found in the 7-day period.

Experimental infection of humans with strains of C. trachomatis isolated from patients with trachoma, inclusion conjunctivitis, or ophthalmia neonatorum produced acute, self-limiting disease with no scarring or pannus. When a subject with previous exposure to $C$. trachomatis was reinoculated more severe disease resulted, with the development of pannus. ${ }^{11}$

Wang and Grayston ${ }^{12}$ found that in Taiwan monkeys infected with various strains of C. trachomatis primary ocular infection produced acute, selflimiting disease, and pannus developed only in animals with previous exposure to $C$. trachomatis.

Field studies in areas of Iran with a high prevalence of trachoma have shown that severe, active disease is common among children, who rarely have blinding complications. These complications are much commoner in adults, who rarely have active disease. ${ }^{13}$ Further studies by Darougar et al. (unpublished observations) have shown that mothers in large families with several young children have a higher incidence of both active disease and blinding complications. These observations on naturally occurring disease show that the blinding lesions of trachoma are late events found when there is a high rate of reinfection.

Our guinea-pig experiments have shown that repeated reinfection produced severe delayed-type hypersensitivity reactions which were followed by the development of severe chronic disease and pannus, scarring, and lid deformities. We therefore suggest that in man the development of blinding hyperendemic trachoma is due to a high rate of reinfection combined with delayed-type hypersensitivity.

We thank Professors Barrie Jones and John Turk for helpful discussions, and the Medical Research Council and an anonymous donor for financial support.

\section{References}

${ }^{1}$ Murray ES. Guinea-pig inclusion conjunctivitis virus. I Isolation and identification as a member of the psittacosislymphogranuloma-trachoma group. J Infect Dis 1964; 114: 1-12.

${ }^{2}$ Kazdan JJ, Schachter J, Okumoto M. Inclusion conjunctivitis in the guinea-pig. Am J Ophthalmol 1967; 64: 116-124. ${ }^{3}$ Monnickendam MA, Darougar S, Treharne JD, Tilbury AM. Guinea-pig inclusion conjunctivitis as a model for the study of trachoma; clinical, microbiological, serological and cytological studies of primary infection. $\mathrm{Br} J$ Ophthalmol 1980; 64: 279-283.

${ }^{4}$ Murray ES, Radcliffe FT. Immunologic studies in guineapigs with guinea-pig inclusion conjunctivitis (GPIC) Bedsonia. Am J Ophthalmol 1967; 63: 1263-1269.

${ }^{5}$ Ahmad A, Dawson CR, Yoneda C, Togni B, Schachter J. Resistance to re-infection with a chlamydial agent (Guineapig inclusion conjunctivitis agent). Invest Ophthalmol Visual Sci 1977; 16: 549-553.

${ }^{6}$ Dawson C, Jawetz E, Hanna L, Rose L, Wood TR, Thygeson, P. Experimental inclusion conjunctivitis in man. II Partial resistance to re-infection. Am J Epidemiol 1966; 84: 411-425.

'Fraser CEO, McComb DE, Murray ES, MacDonald AB. Immunity to chlamydial infections of the eye. IV Immunity in owl monkeys to re-infection with trachoma. Arch Ophthalmol 1975; 93: 518-21.

${ }^{8}$ Watson RR, Mull JD, MacDonald AB, Thompson SE, Bear SE. Immunity to chlamydial infections of the eye. II Studies of passively transferred serum antibody in resistance to infection with guinea-pig inclusion conjunctivitis. Infect Immun 1973; 7: 597-599.

${ }^{9}$ Watson RR, MacDonald AB, Murray ES, Modabber FZ. Immunity to chlamydial infections of the eye. III Presence and duration of delayed hypersensitivity to guinea-pig inclusion conjunctivitis. J Immunol 1973; 111 : 618-623.

${ }^{10}$ Hanna L. Immunofluorescence in chronic TRIC infections of American Indians and Tunisians: influence of trauma on results of tests. In: Nichols RL, ed. Trachoma and Related Disorders Caused by Chlamydial Agents, International Congress Series No. 223. Amsterdam, London, Princeton: Excerpta Medica 1971; 461-468.

11Jones BR, Collier LH. Inoculation of man with inclusion blennorrhea virus. Ann NY Acad Sci 1962; 98: 212-226.

${ }^{12}$ Wang S-P, Grayston JT. Pannus with experimental trachoma and inclusion conjunctivitis agent infection of Taiwan monkeys. Am J Ophthalmol 1967; 63: 1133-1145.

${ }^{13}$ Jones BR. The prevention of blindness from trachoma. Trans Ophthalmol Soc UK 1975; 95: 16-33. 